\title{
Soft Tissue Sarcomas: An Overview on Histomorphology
}

\author{
Shameera Begum ${ }^{1}$, Shanmugasamy Kathirvelu ${ }^{2}$, Anandraj Vaithy ${ }^{3}$, Sowmya Srinivasan ${ }^{4}$
}

\begin{abstract}
Background: Soft tissue sarcomas (STS) comprise a diverse group of rare malignancies that arise from connective tissues. The natural course of STS is unpredictable and aggressive if not diagnosed at an early stage.

Discussion: The subclassification of these tumors is important for prognosis and clinical management of patients. In the present review, we discuss the histomorphologic features of STS and its subtypes based on 2013 World Health Organization classification and throw light on the incidence and presentation of STS.

Conclusion: The systematic approach to morphologic assessment of STS outlined in this review will lead to narrowing of differential diagnoses and pave way for effective diagnosis and implementation of treatment strategies.

Keywords: Classification of soft tissue sarcomas, Histomorphology, Malignant soft tissue tumors, Sarcomas.

Annals of SBV (2019): 10.5005/jp-journals-10085-8114
\end{abstract}

\section{INTRODUCTION}

Soft tissue sarcomas (STSs) are tumors arising from mesodermal portion of the embryo that develops into connective and skeletal tissues. They are uncommon tumors comprising less than $1 \%$ of all malignant tumors. The overall annual incidence of STS is about five cases per 100,000 population. ${ }^{1}$ Although rare, they are life threatening and cause significant diagnostic and therapeutic challenges.

Soft tissue sarcomas can occur at any age and are most common among middle-aged and older adults. They are relatively common in children and young adults constituting $7-10 \%$ of pediatric malignancies. ${ }^{2}$ Most of them are known to arise from retroperitoneum, trunk, and extremities.

The exact etiology of STS is unknown. While few tumors are said to have genetic association such as $10 \%$ lifetime risk of malignant peripheral nerve sheath tumors in individuals with familial neurofibromatosis, majority arise de novo with contributions by environmental factors, irradiation, viral infections (human herpes virus 8 ), and immunodeficiency states. ${ }^{2}$ Soft tissue sarcomas present as painless masses in general, and they are usually large at the time of presentation. Metastasis is in the hematogenous route, and the most common site is lungs.

Magnetic resonance imaging is the investigation of choice due to multiplanar imaging and better tissue discrimination. Final diagnosis is by histopathological examination, as it allows differentiation of benign from malignant cases and in cases of malignancy reports the histologic grade and subtype of sarcoma. ${ }^{3}$

Treatment of STS warrants a multidisciplinary approach. High-grade sarcomas are treated with excision and postoperative radiotherapy. Major advances in the field of immunohistochemistry, cytogenetics, and molecular genetics have led to significant changes in the diagnosis, clinical management, and prognosis of STS.

According to 2013 World Health Organization (WHO) classification, STSs are organized into the following nine groups: adipocytic, fibroblastic/myofibroblastic, the so-called fibrohistiocytic, perivascular, vascular, smooth muscle, skeletal muscle, chondro-osseous tumors, tumors of uncertain differentiation, and undifferentiated sarcomas. The most common
${ }^{1-4}$ Department of Pathology, Mahatma Gandhi Medical College and Research Institute, Pillayarkuppam, Puducherry, India

Corresponding Author: Shanmugasamy Kathirvelu, Department of Pathology, Mahatma Gandhi Medical College and Research Institute, Pillayarkuppam, Puducherry, India, Phone: +91 9487920242, e-mail: shanmugasamyk@mgmcri.ac.in

How to cite this article: Begum S, Kathirvelu S, Vaithy A, Srinivasan S. Soft Tissue Sarcomas: An Overview on Histomorphology. Ann SBV 2019;8(2):45-50.

Source of support: Nil

Conflict of interest: None

histologic subtypes in adults are undifferentiated sarcomas, liposarcoma (LPS), synovial sarcoma, leiomyosarcoma, and malignant peripheral nerve sheath tumor.

In the present review, we discuss the histomorphologic features of STS and its subtypes based on 2013 WHO classification and shed light on the incidence and presentation of STS.

\section{Histomorphological Features of STS}

\section{Adipocytic Tumors}

\section{Liposarcoma}

Liposarcoma is the most common subtype of STS, constituting $20 \%$ of all adult STS. There are four histological subtypes based on clinicopathological characteristics: well-differentiated LPS, myxoid LPS, dedifferentiated LPS, and pleomorphic LPS.

\section{Well-differentiated LPS/Atypical Lipomatous Tumor}

It occurs in adults from fifth to eighth decade with common presentation in the thigh and retroperitoneum. Tumor is grossly lobulated with yellow to white external surface and cut surface that is soft to firm. It is composed of sheets of mature adipocytes showing significant pleomorphism and minimal focal nuclear atypia. Presence of scattered multinucleate and hyperchromatic stromal cells with lipoblasts of varying number contribute to the diagnosis. The rate of recurrence is high in patients with retroperitoneal well-differentiated LPS (Fig. 1). ${ }^{1}$ 


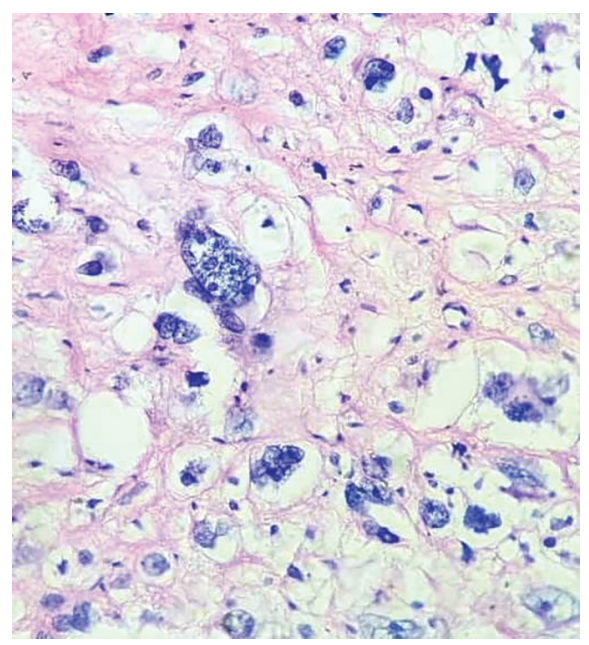

Fig. 1: Liposarcoma showing pleomorphic lipoblasts and atypical mitoses (H\&E, 40X)

\section{Dedifferentiated LPS}

Dedifferentiated LPS presents as large yellow multinodular mass containing solid, gray-tan dedifferentiated areas with necrosis. Microscopy shows areas of well-differentiated LPS with transition to dedifferentiated non-lipogenic sarcoma. ${ }^{4}$ Lipoblastic differentiation within the dedifferentiated component is reported which is termed as dedifferentiated LPS with "homologous lipoblastic differentiation" or "pleomorphic LPS-like features."

Dedifferentiated LPS is overly aggressive, with very high metastatic potential and resistance to chemotherapy and radiation for metastatic cases. ${ }^{1}$

\section{Myxoid LPS}

Myxoid LPS constitutes $30 \%$ of all LPS with peak occurrence at 30-40 years occurring in the extremities. ${ }^{6}$

Grossly, they are circumscribed, multinodular tumors having a gelatinous cut surface. This tumor is composed of round to oval uniform primitive mesenchymal cells admixed with small lipoblasts. The stroma is myxoid with characteristic chicken wire arborizing vascular pattern. ${ }^{6}$

High-grade tumors have round cells without myxoid stroma termed round cell LPS. However, as round cell LPS shares same translocations, the term "round cell liposarcoma" has been removed from the recent WHO classification (2013). ${ }^{5}$

\section{Pleomorphic LPS}

Pleomorphic LPS comprises $5 \%$ of all LPS. It is a high-grade pleomorphic sarcoma composed of varying numbers of pleomorphic lipoblasts. The tumor occurs in the extremities and is seen in adults aged over 40 years. ${ }^{6}$

\section{Fibroblastic/Myofibroblastic Tumors}

\section{Fibrosarcoma}

\section{Adult Fibrosarcoma}

Tumor commonly involves the extremities and trunk and occurs in third to fifth decade of life with a male preponderance. ${ }^{6}$

On gross examination, it is observed as a solitary, circumscribed fleshy tumor with white to tan cut surface. Hemorrhage and necrosis can be seen. ${ }^{6}$
On microscopy, the tumor comprises of spindle-shaped cells arranged in a characteristic herringbone architecture. Areas with storiform pattern may be seen. Individual cells have hyperchromatic nuclei with tapered ends and scant cytoplasm. Collagen in the stroma varies from intercellular delicate network to hypocellular areas with diffuse sclerosis. Tumors that are deep seated tend to be more aggressive. ${ }^{7}$

\section{Myxofibrosarcoma}

Myxofibrosarcoma is more common among elderly males. The site is of occurrence is extremities. ${ }^{8}$

On gross examination, a multinodular gray-white tumor with myxoid appearance is the common observed. On microscopy, low-grade tumors are hypocellular composed of few singly scattered plump spindle cells having eosinophilic cytoplasm and hyperchromatic enlarged nuclei with myxoid stroma. The presence of prominent, curvilinear thin-walled blood vessels surrounded by tumor cells is a characteristic feature. ${ }^{9}$

High-grade neoplasms reveal large solid sheets of spindle cells showing pleomorphism and atypical mitoses with hemorrhagic and necrotic areas. ${ }^{6,9}$

\section{Low-grade Fibromyxoid Sarcoma}

This tumor is commonly seen in the extremities of younger patients. Although the tumor shows bland morphology, there is a significant risk of distant metastasis. On microscopy, it shows highly collagenized, hypocellular areas along with more cellular myxoid areas. Areas of tumor are arranged in whorled pattern, and short fascicles are seen. ${ }^{9}$

\section{Sclerosing Epithelioid Fibrosarcoma}

It is a rare variant of fibrosarcoma with characteristic epithelioid tumor cells embedded in a hyalinized and fibrotic stroma. ${ }^{10}$

\section{So-called Fibrohistiocytic Tumors}

\section{Current Concepts on Malignant Fibrous Histiocytoma}

Malignant fibrous histiocytoma was first introduced by Kauffman and Stout in 1961 and described malignant fibrous histiocytoma (MFH) as a malignant tumor of histiocytic origin with a storiform pattern. By 1977, it was the most common form of adult STS. ${ }^{11}$ This aggressive tumor is poorly characterized on a molecular basis due to overt lack of expression of phenotypic markers in the tumor cells. Malignant fibrous histiocytoma represents a final common pathway of tumors progressing to undifferentiation. ${ }^{12}$ In 2013, the WHO declassified MFH, and the term is obsolete and is replaced by the term "undifferentiated high-grade pleomorphic sarcoma." ${ }^{15}$

Tumor presents as rapidly growing mass over extremities and retroperitoneum in adults older than 40 years. Tumor size may vary from 15 to $20 \mathrm{~cm}$ with tan-white, fleshy to fibrous cut surface with hemorrhage and necrosis. The three histologic subtypes of MFH in 2002 WHO classification are pleomorphic MFH, giant cell MFH, and inflammatory MFH. ${ }^{6}$

\section{Smooth Muscle Tumors Leiomyosarcoma}

Leiomyosarcoma (LMS) arising from smooth muscle connective tissue is comparatively less common and accounts for $10-15 \%$ of sarcomas. It most commonly occurs in the retroperitoneum. ${ }^{13}$ 
Macroscopy shows a fleshy gray-white to tan mass with a whorled appearance which may not be very evident. Microscopically, the tumor shows relatively uniform proliferation of spindle cells in fascicles having cigar-shaped nuclei and bright eosinophilic cytoplasm. Focal areas of storiform or hemangiopericytoma-like arrangement can be seen. The tumor is usually compactly cellular with focal myxoid change and fibrosis. ${ }^{14}$

\section{Skeletal Muscle Tumors Rhabdomyosarcoma \\ Embryonal Rhabdomyosarcoma}

Embryonal rhabdomyosarcoma (RMS) arises from undifferentiated mesoderm and typically affects children younger than 10 years with slight male predominance. Approximately $9 \%$ of these tumors arise within the skeletal muscles of the limbs, and histologically, they resemble developing striated muscles. ${ }^{6}$

Grossly, embryonal RMS is poorly circumscribed, fleshy gray-tan masses. Microscopy shows small rounded or spindleshaped primitive cells exhibiting features of myogenesis, namely, rhabdomyoblasts. As the cells differentiate, cross striations may be seen, and they acquire more eosinophilic and elongated cytoplasm classically described as "strap," "tadpole," or "spider" cells. ${ }^{6}$

\section{Alveolar RMS}

This aggressive tumor is common in children and young adults and present as rapidly enlarging masses in the peripheral extremities. ${ }^{15}$ Histologically, the tumor shows small blue round cells attached to connective tissue septa with myoblastic differentiation and arranged in alveolar pattern. Partial detachment of cells from the fibrous septa which is a formalin fixation-induced artifact gives the tumor its characteristic alveolar appearance. ${ }^{6,15}$

\section{Pleomorphic RMS}

Pleomorphic RMS occurs exclusively in the deep tissues of the lower extermities in adult men in the fifth or sixth decade of life. ${ }^{16}$ Tumor consists of bizarre round, polygonal, or spindle cells with skeletal muscle differentiation. Tumor lacks embryonal or alveolar component.

\section{Spindle Cell/Sclerosing RMS (S-Sc RMS)}

This is an uncommon RMS recently recognized by the WHO in 2013. Spindle cell/sclerosing RMS constitutes 5-13\% of RMS cases, and it can develop in pediatric or adult population. The tumor most commonly occurs in the head and neck region and has an aggressive clinical course with poor prognosis. Microscopy shows varying number of RMS tumor cells embedded in a sclerotic stroma. ${ }^{17}$

\section{VASCULAR TUMORS \\ Angiosarcoma}

Angiosarcomas are rare malignancies of endothelial origin. Majority of them develop as cutaneous tumors. They commonly occur within the muscles of the lower extremities (40\%), upper extremities, trunk, and head and neck. ${ }^{18}$

Grossly, they are multinodular masses with areas of hemorrhage. Size ranges from few to many centimeters in dimension. Angiosarcomas have varied histologic appearance. In general, they have both epithelioid and spindled appearance with the former being more common. The presentation can

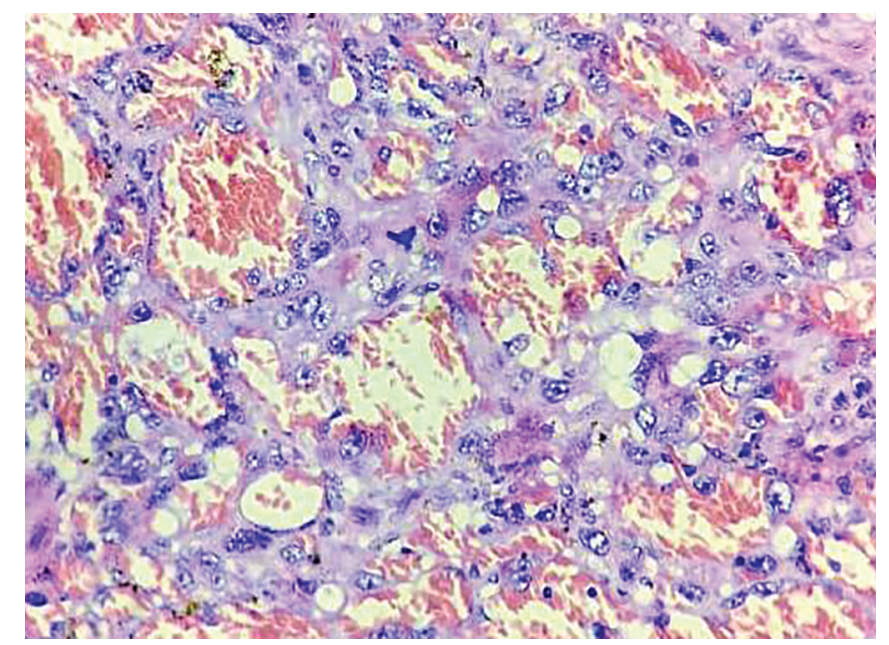

Fig. 2: Epithelioid angiosarcoma showing epithelioid cells with abundant eosinophilic cytoplasm and vesicular nuclei. Atypical mitoses and extensive areas of hemorrhage are seen (H\&E, 40X)

vary from that resembling Kaposi sarcoma to undifferentiated sarcomas. As the tumor has extreme microscopic appearances, it warrants the help of immunohistochemistry (IHC) studies to make a confirmatory diagnosis (Fig. 2). ${ }^{6}$

Epithelioid angiosarcoma is a well-recognized subtype of angiosarcoma, with malignant epithelioid cells being the predominant cytomorphological picture. ${ }^{6}$

\section{Epithelioid Hemangioendothelioma}

Epithelioid hemangioendothelioma is an angiocentric vascular tumor that usually arises from a small vein in the superficial or deep soft tissues of the extremities. It is composed of epithelioid tumor cells arranged in cords, strands, and occasionally in nests in a characteristic myxohyaline stroma. ${ }^{4}$

\section{Chondro-osseous Tumors}

\section{Mesenchymal Chondrosarcoma}

Mesenchymal chondrosarcoma is an exceedingly rare malignant tumor characterized by a bimorphic pattern composed of areas of primitive appearing small round cells and islands of mature hyaline cartilage. The peak incidence is in the second and third decades of life and has a female predominance. The jaw bones, ribs, and thigh are the most commonly involved anatomical sites.

\section{Nerve Sheath Tumors \\ Malignant Peripheral Nerve Sheath Tumor}

Over $5-10 \%$ of sarcomas are malignant peripheral nerve sheath tumors (MPNSTs) occurring in late adolescence and early adulthood. ${ }^{6}$ Malignant peripheral nerve sheath tumors occur in $5-42 \%$ of patients with multiple neurofibromatosis (NF). ${ }^{19}$ Malignant spindled tumors in patients with NF1 should be considered MPNST until proven otherwise. These tumors are typical large with fleshy mucoid cut surface with hemorrhage and necrosis. Histology shows areas of alternating cellularity, fascicles, palisades, whorls, and rosette-like arrangements with subendothelial accentuation of tumor cells. Also seen are large areas of geographic-like necrosis. ${ }^{20}$ 


\section{Epithelioid MPNST}

Epithelioid MPNST accounts for 5\% of MPNST. It is rare and has infrequent association with NF1 and occasional origin from a schwannoma. ${ }^{21}$

\section{Malignant Triton Tumor}

Malignant triton tumor is a malignant peripheral nerve sheath tumor with heterologous rhabdomyoblastic differentiation. The unusual name "triton" was derived from an experiment, where sciatic nerve tissue of the triton salamander was stimulated to grow a neoplasm with skeletal muscle components. Common sites of occurrence are head, neck, extremities, and trunk. It has an aggressive biological behaviour. ${ }^{22,23}$

\section{Malignant Granular Cell Tumor}

It is a rare, aggressive neural tumor and represents $1-2 \%$ of all granular cell tumors. Tumor commonly presents in the lower extremities unlike benign granular cell tumors that occur in the head and neck and tongue. ${ }^{24}$

\section{Ectomesenchymoma}

Also called malignant ectomesenchymoma, it is a rare, rapidly growing tumor of the nervous system or soft tissue that mainly occurs in children. Reported sites are abdomen, head and neck, perineum, scrotum, and limbs. ${ }^{25}$

\section{Tumors of Uncertain Differentiation Synovial Sarcoma}

Synovial sarcoma is one of the most common soft tissue sarcoma accounting for $5.3 \%$ of all STSs. ${ }^{26}$ It is common in the age-group of 15-40 years with predilection for males. ${ }^{27}$ They occur in the extremities close to large joints and are also known to occur in the retroperitoneum (Fig. 3). ${ }^{27,28}$

Grossly, they are well delineated with a fibrous pseudocapsule and a soft, tan, and homogeneous cut surface. Histologically, the tumor shows two morphologically distinct cell population forming a biphasic pattern. Epithelial cells and spindle cells along with areas of hyalinization, myxoid changes, and calcifications are noted. ${ }^{27,29}$

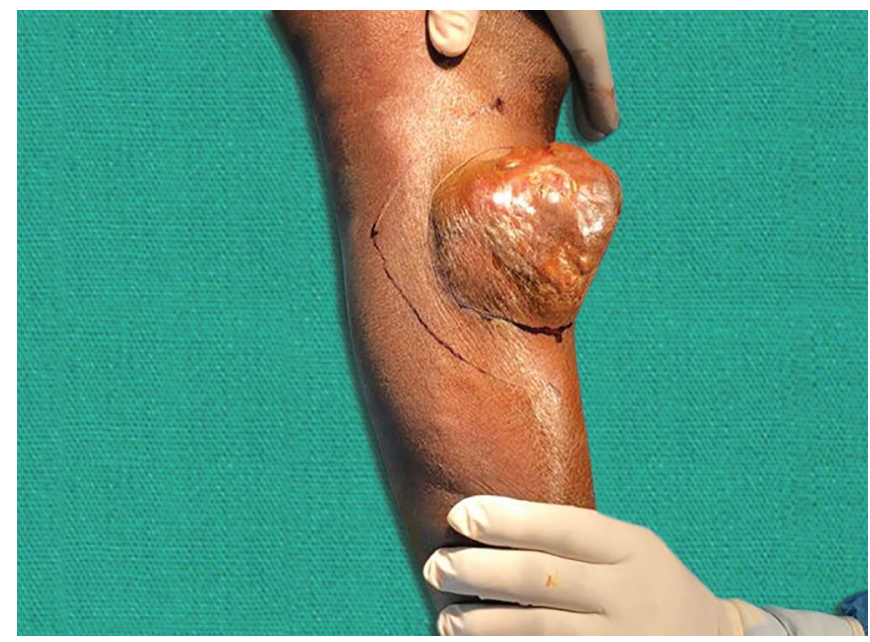

Fig. 3: Clinical picture of synovial sarcoma of leg

\section{Epithelioid Sarcoma}

Epithelioid sarcomas are rare mesenchymal tumors of unknown differentiation accounting for less than $1 \%$ of STSs. They are usually slow growing and occur in young adult men, predominantly in extremities. Histologically, the tumor is characterized by epithelioid cells that aggregate in nodule and undergo central necrosis. Individual tumor cells are bland, polygonal cells with eosinophilic cytoplasm and peripheral spindling. ${ }^{30}$

\section{Alveolar STS}

It is a rare neoplasm forming only $0.5 \%$ of STSs. ${ }^{26}$ It usually presents in adolescents and young adults, mostly in females. The common site occurrence is lower extremities, especially in the anterior aspect of thigh. Distant metastasis of the lungs is a common presentation. ${ }^{31}$

On gross examination, they are soft, friable, and ill-defined with areas of hemorrhage and necrosis on cut section. Histology shows tumor that is multilobulated and arranged in organoid, alveolar, or pseudo-alveolar pattern due to loss of cell cohesion within the tumor nests. Individual tumor cells are rounded or polygonal cells having abundant eosinophilic granular cytoplasm. ${ }^{31}$

\section{Clear Cell Sarcoma of Soft Tissue}

Clear cell sarcoma of tendons and aponeuroses typically occurs in foot and ankle of children and adolescents. Grossly, the tumor is well circumscribed with a gray or white cut surface. Microscopy shows tumor cells that are fusiform or cuboidal arranged in nests and fascicles with abundant to clear cytoplasm, vesicular nuclei, and prominent nucleoli. ${ }^{6}$

\section{Extraskeletal Myxoid Chondrosarcoma}

This is a rare low-grade STS with a predilection for a primary site in the extremities, typically presenting during the fifth and sixth decades of life. Histologically, tumor is lobulated with and malignant chondroblast-like cells arranged in cords, strands, or clusters in an abundant myxoid stroma ${ }^{32}$

\section{Extraskeletal Ewing Sarcoma}

It is a rare aggressive malignant tumor occurring in adolescents and young adults between 10 and 30 years of age with a male predilection. The most common sites of occurrence are chest wall, paravertebral region, lower extremities, retroperitoneum, and gluteal region. ${ }^{33}$

They are lobulated masses usually measuring less than $10 \mathrm{~cm}$. Microscopically, the tumor is composed of monomorphous sheets of uniformly packed cells with small round nuclei, having finely granular chromatin, and scant clear cytoplasm. Mitotic figures are scantly present. Cytoplasm of tumor cells contains PAS-positive glycogen. Homer-Wright rosettes are also seen. ${ }^{6}$

\section{Desmoplastic Small Round Cell Tumor}

Desmoplastic small round cell tumor is a rare tumor with striking male predilection. The tumor is composed of nests of small round tumor cells within a desmoplastic stroma containing spindleshaped myofibroblasts. Majority of patients develop tumor in the abdominal cavity, with the most frequent site being the retroperitoneum. ${ }^{4}$

\section{Extra Renal Rhabdoid Tumor}

Soft tissue rhabdoid tumor occurs in infants and children. It is characterized by tumor cells with large nuclei and abundant, 


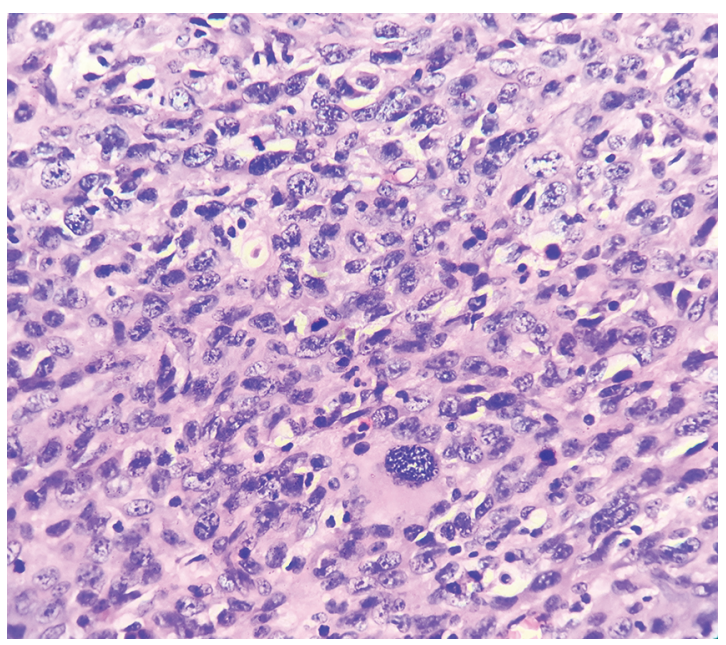

Fig.4: Undifferentiated pleomorphic sarcoma showing bizarre cells and atypical mitoses (H\&E, 40X)

eccentric cytoplasm with prominent eosinophilic, cytoplasmic "inclusions." The tumors arise in the neck and paraspinal regions. ${ }^{4}$

\section{Undifferentiated/Unclassified Sarcomas}

Undifferentiated spindle cell sarcoma, undifferentiated pleomorphic sarcoma (Fig. 4), undifferentiated round cell sarcoma, undifferentiated epithelioid sarcoma, and undifferentiated sarcoma, not otherwise specified, are subtypes under this category.

\section{Malignant Gastrointestinal Stromal Tumors}

Gastrointestinal stromal tumors (GISTs) were included for the first time in 2013 WHO classification of soft tissue tumors. ${ }^{5}$ Twenty-five percent of GIST are malignant. Malignant GISTs are typically large, well-circumscribed, heterogeneous tumors with central necrosis. The tumors usually arise in the wall of the small bowel or stomach. Malignant GISTs have extremely poor prognosis and are resistant to radiotherapy. ${ }^{34}$

\section{Grading of STSS}

The staging of STS largely depends on its histological grade. The most widely used STS grading systems are The French Federation Nationale des Centres de Lutte Contre le Cancer (FNCLCC) grading system and National Cancer Institute (NCI). Both the systems have 3 grades and are based on mitotic activity, necrosis, and differentiation which are known to correlate well with the prognosis. ${ }^{35}$ However, the $\mathrm{NCl}$ system, in addition, requires the quantification of cellularity and pleomorphism for few subtypes of sarcomas which is difficult to determine objectively. The FNCLCC system is easier to use and appears slightly better in predicting prognosis (Table 1). ${ }^{35}$

\section{Conclusion}

Over the past few decades, our knowledge on the presentation of STSs has showed little progress compared to other malignancies. Soft tissue sarcomas, being aggressive tumors, have acquired many newer entities based on the origin of derivation, thereby warranting regular update on the diagnosis by routine histomorphology. Several morphologic and systematic pattern approaches to STS outlined in this review will lead to narrowing of differential diagnoses and pave way for effective diagnosis and treatment strategies to move the field forward.
Table 1: FNCLCC histological grading of soft tissue sarcomas ${ }^{35}$

\begin{tabular}{lll}
\hline $\begin{array}{l}\text { Tumor differentiation } \\
\text { 1: Sarcomas that closely }\end{array}$ & Necrosis & $\begin{array}{l}\text { Mitotic count }(\text { per } \\
\text { 10 high power fields) }\end{array}$ \\
$\begin{array}{l}\text { resemble normal mature } \\
\text { mesenchymal tissue }\end{array}$ & $1: 0-9$ mitoses \\
$\begin{array}{l}\text { 2: Sarcomas of definite } \\
\text { histologic type }\end{array}$ & $1:<50 \%$ necrosis & $2: 10-19$ mitoses \\
$\begin{array}{l}\text { 3: Embryonal and undif- } \\
\text { ferentiated sarcomas, } \\
\text { synovial sarcoma, and } \\
\text { sarcomas of uncertain } \\
\text { differentiation }\end{array}$ & $2: 50 \%$ necrosis & $3:>19$ mitoses \\
\hline
\end{tabular}

Histological grade: grade I-total score 2 or 3/grade II —-total score 4 or 5/ grade III-total score 6,7 or 8

\section{References}

1. Hoang NT, Acevedo LA, Mann MJ, Tolani B. A review of soft-tissue sarcomas: translation of biological advances into treatment measures. Cancer Manag Res 2018;10:1089-1114. DOI: 10.2147/CMAR. S159641.

2. Grimer R, Judson I, Peake D, Seddon B. Guidelines for the management of soft tissue sarcomas. Sarcoma 2010;2010:506182. DOI: 10.1155/2010/506182.

3. van Vliet M, Kliffen M, Krestin GP, van Dijke CF. Soft tissue sarcomas at a glance: clinical, histological, and MR imaging features of malignant extremity soft tissue tumors. Eur Radiol 2009;19(6):1499-1511. DOI: 10.1007/s00330-008-1292-3.

4. Fletcher CDM, Bridge JA, Hogendoorn PCW, Mertens F, ed. WHO Classification of Soft Tissue and Bone Tumors. 4th ed., Geneva, Switzerland: WHO Press; 2013.

5. Doyle LA. Sarcoma classification: An update based on the 2013 World Health Organization Classification of Tumors of Soft Tissue and Bone: WHO Update of Sarcoma Classification. Cancer 2014;120(12):17631774. DOI: 10.1002/cncr.28657.

6. World Health Organisation Classification of Tumours. In: Fletcher CDM, Unni KK, Mertens F, ed. Pathology and Genetics of Tumours of Soft Tissue. Lyon: IARC Press; 2002. pp. 12-224.

7. Augsburger D, Nelson PJ, Kalinski T, Udelnow A, Knösel T, Hofstetter $M$, et al. Current diagnostics and treatment of fibrosarcoma perspectives for future therapeutic targets and strategies. Oncotarget 2017;8(61):104638-104653. DOI: 10.18632/oncotarget.20136.

8. Castronovo C, Arrese JE, Quatresooz P, Nikkels AF. Myxofibrosarcoma: A Diagnostic Pitfall. Rare Tumors 2013;5(2):60-61. DOI: 10.4081/ rt.2013.e15.

9. Oda Y, Takahira T, KawaguchiK, Yamamoto H, Tamiya S, Matsuda S, et al. Low-grade fibromyxoid sarcoma versus low-grade myxofibrosarcoma in the extremities and trunk. A comparison of clinicopathological and immunohistochemical features. Histopathology 2004;45(1):29-38. DOI: 10.1111/j.1365-2559.2004.01886.x.

10. Antonescu CR, Rosenblum MK, Pereira P, Nascimento AG, Woodruff JM. Sclerosing epithelioid fibrosarcoma: a study of 16 cases and confirmation of a clinicopathologically distinct tumor. Am J Surg Pathol 2001;25(6):699-709. DOI: 10.1097/00000478-20010600000001.

11. Akerman M. Malignant fibrous histiocytoma--the commonest soft tissue sarcoma or a nonexistent entity? Acta Orthop Scand Suppl 1997;273:41-46. DOI: 10.1080/17453674.1997.11744701.

12. Gazziola C, Cordani N, Wasserman B, Carta S, Colombatti A, Perris R. Malignant fibrous histiocytoma: a proposed cellular origin and identification of its characterizing gene transcripts. Int J Oncol 2003;23(2):343-351. DOI: 10.3892/ijo.23.2.343.

13. Paal E, Miettinen M. Retroperitoneal leiomyomas: a clinicopathologic and immunohistochemical study of 56 cases with a comparison to 
retroperitoneal leiomyosarcomas. Am J Surg Pathol 2001;25(11):13551363. DOI: 10.1097/00000478-200111000-00002.

14. Rajani B, Smith TA, Reith JD, Goldblum JR. Retroperitoneal leiomyosarcomas unassociated with the gastrointestinal tract: a clinicopathologic analysis of 17 cases. Mod Pathol 1999;12(1):21-28.

15. Enterline HT, Horn RC. Alveolar rhabdomyosarcoma. A distinctive tumor type. Am J Clin Pathol 1958;29(4):356-366. DOI: 10.1093/ ajcp/29.4.356.

16. Furlong MA, Mentzel T, Fanburg-Smith JC. Pleomorphic rhabdomyosarcoma in adults: a clinicopathologic study of 38 cases with emphasis on morphologic variants and recent skeletal musclespecific markers. Mod Pathol 2001;14(6):595-603. DOI: 10.1038/ modpathol.3880357.

17. Smith $\mathrm{MH}$, Atherton $\mathrm{D}$, Reith JD, Islam NM, Bhattacharyya I, Cohen DM. Rhabdomyosarcoma, spindle cell/sclerosing variant: a clinical and histopathological examination of this rare variant with three new cases from the oral cavity. Head Neck Pathol 2017;11(4):494-500. DOI: 10.1007/s12105-017-0818-x.

18. Hart J,Mandavilli S. Epithelioid angiosarcoma: a brief diagnostic review and differential diagnosis. Arch Pathol Lab Med 2011;135(2):268-272. DOI: 10.1043/1543-2165-135.2.268.

19. Rekhi B, Ingle A, Kumar R, DeSouza MA, Dikshit R, Jambhekar NA Malignant peripheral nerve sheath tumors: clinicopathological profile of 63 cases diagnosed at a tertiary cancer referral center in Mumbai, India. Indian J Pathol Microbiol 2010;53(4):611-618. DOI: 10.4103/0377-4929.71998.

20. Rekhi B, Jambhekar NA. Malignant transformation in a hybrid schwannoma/perineurioma: addition to the spectrum of a malignant peripheral nerve sheath tumor. Indian J Pathol Microbiol 2011;54(4):825-828. DOI: 10.4103/0377-4929.91542.

21. Jo VY, Fletcher CDM. Epithelioid malignant peripheral nerve sheath tumor: clinicopathologic analysis of 63 cases. Am J Surg Pathol 2015;39(5):673-682. DOI: 10.1097/PAS.0000000000000379.

22. Tripathy K, Mallik R, Mishra A, Misra D, Rout N, Nayak P, et al. A rare malignant triton tumor. Case Rep Neurol 2010;2(2):69-73. DOI: 10.1159/000315621.

23. Li G, Liu C, Liu Y, Xu F, Su Z, Wang Y, et al. Analysis of clinical features and prognosis of malignant triton tumor: a report of two cases and literature review. Oncol Lett 2015;10(6):3551-3556. DOI: 10.3892/ ol.2015.3762.
24. Gupta N, Sanchety N, Verma PS, Verma G. Malignant granular cell tumor of the breast; literature review. Indian J Pathol Microbiol 2015;58(2):238. DOI: 10.4103/0377-4929.155330.

25. Dantonello TM, Leuschner I, Vokuhl C, Gfroerer S, Schuck A, Kube S, et al. Malignant ectomesenchymoma in children and adolescents: report from the Cooperative Weichteilsarkom Studiengruppe (CWS). Pediatr Blood Cancer 2013;60(2):224-229. DOI: 10.1002/ pbc.24174.

26. Kransdorf MJ. Malignant soft-tissue tumors in a large referral population: distribution of diagnoses by age, sex, and location. AJR Am J Roentgenol 1995;164(1):129-134. DOI: 10.2214/ajr.164.1.7998525.

27. Stacchiotti S, Van Tine BA. Synovial sarcoma: current concepts and future perspectives. J Clin Oncol 2018;36(2):180-187. DOI: 10.1200/ JCO.2017.75.1941.

28. Fisher C, Folpe AL, Hashimoto H, Weiss SW. Intra-abdominal synovial sarcoma: a clinicopathological study. Histopathology 2004;45(3):245253. DOI: 10.1111/j.1365-2559.2004.01950.x.

29. Mohaidat ZM, Saleh A-AA, Al-Gharaibeh S, Yousef IR. Case report: synovial sarcoma of the axilla with brachial plexus involvement. World J Surg Oncol 2018;16(1):166. DOI: 10.1186/s12957-0181466-7.

30. Armah HB, Parwani AV. Epithelioid sarcoma. Arch Pathol Lab Med 2009;133(5):814-819. DOI: 10.1043/1543-2165-133.5.814.

31. Folpe AL, Deyrup AT. Alveolar soft-part sarcoma: a review and update. J Clin Pathol 2006;59(11):1127-1132. DOI: 10.1136/jcp.2005. 031120.

32. Ananthamurthy A, Nisheena R, Rao B, Correa M. Extraskeletal myxoid chondrosarcoma: diagnosis of a rare soft tissue tumor based on fine needle aspiration cytology. J Cytol 2009;26(1):36-38. DOI: 10.4103/0970-9371.54867.

33. Applebaum MA, Worch J, Matthay KK, Goldsby R, Neuhaus J, West DC, et al. Clinical features and outcomes in patients with extraskeletal Ewing sarcoma. Cancer 2011;117(13):3027-3032. DOI: 10.1002/ cncr.25840.

34. Shukla S, Singh SK, Pujani M. Multicentric malignant gastrointestinal stromal tumor. Saudi J Gastroenterol 2009;15(1):45-48. DOI: 10.4103/1319-3767.45055.

35. Coindre J-M. Grading of soft tissue sarcomas: review and update. Arch Pathol Lab Med 2006;130(10):1448-1453. DOI: 10.1043/1543-2165(2006)130[1448:GOSTSR]2.0.CO;2. 